Annali di Matematica pura ed applicata

(IV), Vol. CLXXVIII (2000), pp. 195-224

\title{
Continuous Solutions for a Degenerate Free Boundary Problem $(*)$.
}

\author{
José Miguel Urbano
}

Abstract. - We prove existence of continuous solutions for

$$
\partial_{t}[\gamma(\theta)]-\operatorname{div}\left(|\nabla \theta|^{p-2} \nabla \theta\right) \ni 0, \quad p>2,
$$

where $\gamma$ is a maximal monotone graph, by showing equicontinuity of a sequence of approximate solutions. Relations of this type are models for certain free boundary problems like the Stefan problem with nonlinear diffusion.

\section{1. - Introduction.}

This paper deals with the question of the continuity of the solution of degenerate parabolic equations, with principal part in divergence form, of the type

$$
\partial_{t}[\gamma(\theta)]-\operatorname{div}\left(|\nabla \theta|^{p-2} \nabla \theta\right) \ni 0, \quad p>2,
$$

where $\gamma$ is a maximal monotone graph with a singularity at the origin. The equation is thus degenerate in the space part, due to the vanishing of its modulus of ellipticity $|\nabla \theta|^{p-2}$ at points where $|\nabla \theta|=0$, and singular in the time part since $\left\langle\gamma^{\prime}(0)=\infty »\right.$. We use the word singular with this meaning and not in reference to the principal part singular case corresponding to $1<p<2$, which is not considered here.

An equation of this form occurs as a model for the well known two phase Stefan problem when a nonlinear law of diffusion is considered, $\theta$ being in that case the temperature and $\gamma(\theta)$ the enthalpy. The problem of the continuity of the temperature in the linear diffusion case $(p=2)$ was solved independently by Caffarelli and Evans (cf. [1]) and DiBenedetto (cf. [3]) in the early eighties. The technique developed in [3] is more powerful since it works not only for the Laplacian, whose properties are essential

(*) Entrata in Redazione il 17 dicembre 1998.

Indirizzo dell'A.: Departamento de Matemática da Universidade de Coimbra, Apartado 3008 3000 Coimbra, Portugal. E-mail: jmurb@mat.uc.pt

1991 Mathematics Subject Classification: 35D10, 35K65, 35R35.

Research supported by CMUC-FCT, Praxis XXI and project Praxis/2/2.1/MAT/125/94. 
in the proof of [1], but also for more general principal parts, satisfying appropriate structure assumptions. It covers also the case of equations with lower order terms depending on the temperature, which is relevant when we are taking convection into account.

Concerning the case of nonlinear diffusion, the question of the existence was successfully tackled in [11] by means of a regularization method and the use of an extended weak maximum principle. The very week regularity available in what concerns the time derivative, that only has a meaning as a distribution, prevented us so far from obtaining a uniqueness result. We show here that the problem has at least a continuous solution, namely the one that was obtained in the proof of the existence result as the limit of the sequence of approximate solutions. These are the unique solutions of problems obtained after regularization of the maximal monotone graph. The result is achieved by showing that the equibounded sequence of approximations is also equicontinuous.

The regularity of solutions of degenerate parabolic equations has deserved a considerable amount of attention in recent years and a good reference in what concerns the state of the art is the book by DiBenedetto [6]. Results on the continuity of solutions at a point consist basically in constructing a sequence of nested and shrinking cylinders with vertex at that point, such that the essential oscillation of the function in those cylinders converges to zero when the cylinders shrink to the point. At the basis of the proof is an iteration technique, that is a refinement of the technique by DeGiorgi and Moser (cf. [2], [9] and [8]), based on energy (and logarithmic) estimates for the solution, that in the degenerate case are not homogeneous in the sense that they involve integral norms corresponding to different powers, namely the powers 2 and $p$. The key idea is to look at the equation in its own geometry, i.e., in a geometry dictated by its degenerate structure. This amounts to rescale the standard parabolic cylinders by a factor like

$$
\left(\frac{\omega}{A}\right)^{p-2}, \quad A=2^{s}
$$

where $\omega$ is an upper bound for the oscillation of the solution in the rescalled cylinder. This procedure, which can be called accommodation of the degeneracy, allows one to recover the homogeneity in the energy estimates written over these rescalled cylinders and carry on with the proof. We can say heuristically that the equation behaves in its own geometry like the heat equation.

In the present singular-degenerate case, when showing that the approximate solutions are equicontinuous, a third power (power 1) occurs at the energy estimates, as a consequence of estimating uniformly the regularization of the maximal monotone graph. No rescalling permits the compatibility of the three powers so we use the geometry of the nonsingular case to deal with the degeneracy and pay the price of a dependence on the oscillation in the various constants that are determined along the proof. Owing to this fact we are no longer able to exhibit a modulus of continuity but only to define it implicitly independently of the regularization. This is enough to obtain a continuous solution for the original problem but the Hölder continuity, that holds in the nonsingular case, is lost. 
The article is organized as follows. In the next section we formulate the problem and state the main result concerning the interior equicontinuity of a sequence of approximate solutions. In section 3 we derive the essential tools for the proof: the independent of $\varepsilon$ local energy and logarithmic estimates. The geometric setting is presented in section 4, where we also reduce the proof to the analysis of two alternative cases, that are considered separately in sections 5 and 6 . The proof of the main results is the object of the last section.

We made an effort to keep the article self contained and enjoyable to read without repeating too much of what is already known from the literature. Some duplication of arguments was although inevitable.

\section{Notation.}

- $\Omega$ is a bounded and regular domain in $\mathbb{R}^{N}$, with boundary $\partial \Omega$.

- $Q=\Omega \times(0, T)$, for $T>0$ is the space-time domain with lateral boundary $\Sigma=$ $=\partial \Omega \times(0, T)$ and parabolic boundary $\partial_{p} Q=\Sigma \cup(\Omega \times\{0\})$.

- We use the usual Sobolev spaces and define

$$
V_{0}^{p}(Q)=L^{\infty}\left(0, T ; L^{p}(\Omega)\right) \cap L^{p}\left(0, T ; W_{0}^{1, p}(\Omega)\right)
$$

endowed with the norm

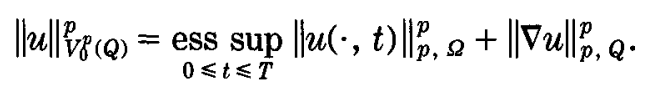

- The characteristic function of a set $A$ is denoted by $\chi_{A}$.

- Given a point $x_{0} \in \mathbb{R}^{N}, K_{\varrho}\left(x_{0}\right)$ denotes the $N$-dimensional cube with centre at $x_{0}$ and wedge $2 \varrho$ :

$$
K_{\varrho}\left(x_{0}\right):=\left\{x \in \mathbb{R}^{N}: \max _{1 \leqslant i \leqslant N}\left|x_{i}-x_{0 i}\right|<\varrho\right\} ;
$$

given a point $\left(x_{0}, t_{0}\right) \in \mathbb{R}^{N+1}$, the cylinder of radius $\varrho$ and height $\tau>0$ is

$$
\left(x_{0}, t_{0}\right)+Q(\tau, \varrho):=K_{\varrho}\left(x_{0}\right) \times\left(t_{0}-\tau, t_{0}\right) .
$$

- Throughout the paper, the letter $C$ denotes a constant that depends only on the data. The same $C$ will be used to denote different constants depending on the data except if this occurs in the same line. In that case we use $C_{1}, C_{2}$, etc.

\section{2. - Statement of the problem and main result.}

In [11] we study a generalized Stefan problem from the point of view of the existence of a solution. The novelty in the problem is the assumption of a nonlinear Fourier law relating the heat flux and the gradient of the renormalized temperature field $\theta$, 
that gives rise to a diffusion term in the equation involving the $p$-Laplacian operator

$$
\Delta_{p} \theta:=\operatorname{div}\left(|\nabla \theta|^{p-2} \nabla \theta\right) .
$$

In the case $p>2$, to which we will be restricted, this renders the equation degenerate since its modulus of ellipticity $|\nabla \theta|^{p-2}$ vanishes at points where $|\nabla \theta|=0$.

We assume the material under consideration occupies a domain $\Omega$, with two phases, a solid phase corresponding to the region $\{\theta<0\}$ and a liquid phase corresponding to the region $\{\theta>0\}$, separated by an interface $\Phi=\{\theta=0\}$, the free boundary. The problem in its strong formulation reads

$$
(\boldsymbol{P}) \begin{cases}\left(\partial_{t}+\boldsymbol{v} \cdot \nabla\right) b(\theta)=\Delta_{p} \theta & \text { in } Q \backslash \Phi=\{\theta<0\} \cup\{\theta>0\} \\ {\left[|\nabla \theta|^{p-2} \nabla \theta\right]_{-}^{+} \cdot \boldsymbol{n}=\lambda(\boldsymbol{w}-\boldsymbol{v}) \cdot \boldsymbol{n}} & \text { on } \Phi=\{\theta=0\} \\ \boldsymbol{\theta}=\theta_{D} & \text { on } \Sigma \\ \theta(0)=\theta_{0} & \text { in } \Omega \times\{0\}\end{cases}
$$

where $\boldsymbol{v}$ is a prescribed velocity field, $b$ a given continuous and increasing function, $n$ the unit normal to $\Phi$, pointing to the solid region, $w$ the velocity of the free boundary and $\lambda=[e]_{-}^{+}>0$ the latent heat of phase transition ( $e$ is the internal energy), with $[.]^{ \pm}$ denoting the jump across $\Phi$.

Following the original ideas of [7] we derive a weak formulation, in which all explicit references to the free boundary are absent, considering the maximal monotone graph $H$ associated with the Heaviside function,

$$
H(s)= \begin{cases}0 & \text { if } s<0 \\ {[0,1]} & \text { if } s=0 \\ 1 & \text { if } s>0\end{cases}
$$

and introducing a new unknown function, the enthalpy $\eta$, such that

$$
\eta \in \gamma(\theta):=b(\theta)+\lambda H(\theta) .
$$

A formal integration by parts against appropriate test functions and the replacement of the initial condition for $\theta$ by a more adequate initial condition for $\eta$, leads to an integral relation that we adopt as definition of weak solution.

We will restrict ourselves to the cases

$$
v=0, \quad b(s) \equiv s, \quad \theta_{D}=0, \quad p>2 .
$$

A more general $b \in C^{0,1}(\mathbb{R})$ satisfying the conditions $b(0)=0$ and $0<b_{*} \leqslant b^{\prime} \leqslant b_{*}$ can be considered; the problem can be reduced to the case $b(s)=s$ in a simple way (see [3]). It is also possible to deal with the case of a convective term depending on the temperature at the expense of a more technical analysis (see [6]). We don't consider it here to focus the attention on the really new difficulty, which is to deal with the singularity in time and the degeneracy in space at the same time. The case of a convective term depending on the enthalpy, like the convective term in [11], is more delicate and is left 
open. As far as we know, not even in the nondegenerate case $(p=2)$ continuity results are available. The existence of weak solutions is obtained in [10], where it is also shown that $\partial_{t} \theta \in L_{\mathrm{loc}}^{2}(Q)$, a regularity result that may be relevant to obtain the continuity of the temperature. The last two restrictions in (1) reflect severe difficulties with which we are unable to deal at the present moment. We will refer to this later in the last section.

With this restrictions in mind, the exact definition of weak solution, the space of test functions being

$\mathscr{T}(Q):=\left\{\xi \in L^{p}\left(0, T ; W_{0}^{1, p}(\Omega)\right): \partial_{t} \xi \in L^{2}(Q), \xi(T)=0\right\}$,

is the following (see [11])

Definition 1. - We say that $(\eta, \theta)$ is a weak solution of problem $(\boldsymbol{P})$, if

$$
\begin{gathered}
\theta \in L^{p}\left(0, T ; W_{0}^{1, p}(\Omega)\right) \cap L^{\infty}(Q) ; \\
\eta \in L^{\infty}(Q) \quad \text { and } \quad \eta \in \gamma(\theta), \quad \text { a.e. in } Q ; \\
-\int_{Q} \eta \partial_{t} \xi+\int_{Q}|\nabla \theta|^{p-2} \nabla \theta \cdot \nabla \xi=\int_{\Omega} \eta_{0} \xi(0), \quad \forall \xi \in \mathscr{T}(Q) .
\end{gathered}
$$

The proof of the existence result in [11], assuming

$$
\eta_{0} \in \gamma\left(\theta_{0}\right) \quad \text { and } \quad\left\|\theta_{0}\right\|_{L^{\infty}(\Omega)} \leqslant M, \quad M>0
$$

consists in a regularization of the maximal monotone graph and some data, leading to approximated problems that are solved by a Galerkin method. The derivation of suitable $a$ priori estimates and the use of monotonicity methods allow us to pass to the limit and obtain a bounded solution to the problem. More precisely, let $0<\varepsilon<1$ and consider the function

$$
\gamma_{\varepsilon}(s)=s+\lambda H_{\varepsilon}(s)
$$

where $H_{\varepsilon}$ is a $\mathcal{C}^{\infty}$-approximation of the Heaviside function, such that

$$
H_{\varepsilon}(s)=0 \quad \text { if } s \leqslant 0, \quad \mathrm{H}_{\varepsilon}(\mathrm{s})=1 \quad \text { if } s \geqslant \varepsilon,
$$

$H_{\varepsilon}^{\prime} \geqslant 0$ and $H_{\varepsilon} \rightarrow H$ uniformly in the compact subsets of $\mathbb{R} \backslash\{0\}$, as $\varepsilon \rightarrow 0$. The function $\gamma_{\varepsilon}$ is bilipschitz and satisfies

$$
1 \leqslant \gamma_{\varepsilon}^{\prime}(s) \leqslant 1+\lambda L_{\varepsilon}, \quad s \in \mathbb{R},
$$

with $L_{\varepsilon} \equiv \mathcal{O}(1 / \varepsilon)$ being the Lipschitz constant of $H_{\varepsilon}$. Its inverse $\beta_{\varepsilon}=\gamma_{\varepsilon}^{-1}$ satisfies

$$
0<\frac{1}{1+\lambda L_{\varepsilon}} \leqslant \beta_{\varepsilon}^{\prime}(s) \leqslant 1, \quad s \in \mathbb{R} .
$$


Taking also a sequence of functions $\theta_{0 \varepsilon} \in W^{1, p}(\Omega)$ such that

$$
\theta_{0 \varepsilon} \rightarrow \theta_{0}, \quad \gamma_{\varepsilon}\left(\theta_{0 \varepsilon}\right) \rightarrow \eta_{0} \text { in } L^{p}(\Omega) \text { and }\left|\theta_{0 \varepsilon}\right| \leqslant M \text {, a.e. in } \Omega
$$

we define the approximated problem as follows $\left(P_{\varepsilon}\right)$ : For each $0<\varepsilon \ll 1$, find a function

$$
\theta_{\varepsilon} \in H^{1}\left(0, T ; L^{2}(\Omega)\right) \cap L^{\infty}\left(0, T ; W_{0}^{1, p}(\Omega)\right) \cap L^{\infty}(Q)
$$

such that

$$
-\int_{Q} \gamma_{\varepsilon}\left(\theta_{\varepsilon}\right) \partial_{t} \xi+\int_{Q}\left|\nabla \theta_{\varepsilon}\right|^{p-2} \nabla \theta_{\varepsilon} \cdot \nabla \xi=\int_{\Omega} \gamma_{\varepsilon}\left(\theta_{0 \varepsilon}\right) \xi(0), \quad \forall \xi \in \mathscr{J}(Q) .
$$

In the presence of the regularity required, equation (4) can be shown to be equivalent to the two conditions: $\theta_{\varepsilon}(0)=\theta_{0 \varepsilon}$ and, for a.e. $t \in(0, T)$,

$$
\int_{\Omega \times\{t\}} \partial_{t}\left[\gamma_{\varepsilon}\left(\theta_{\varepsilon}\right)\right] \varphi+\int_{\Omega \times\{t\}}\left|\nabla \theta_{\varepsilon}\right|^{p-2} \nabla \theta_{\varepsilon} \cdot \nabla \varphi=0, \quad \forall \varphi \in W_{0}^{1, p}(\Omega) .
$$

We show in [11] that this approximated problem has a unique solution and derive enough a priori estimates to pass to the limit and obtain a solution of the original problem with the regularity required in Definition 1, that in addition satisfies an extended weak maximum principle:

$$
\|\theta\|_{L^{\infty}(Q)} \leqslant M
$$

It is clear from the results of [6] that the solutions of the approximated problem are Hölder continuous. In fact, they satisfy in the distribution sense an equation of the type

$$
\partial_{t} u-\operatorname{div} \boldsymbol{a}(x, t, u, \nabla u)=0
$$

with $u=\gamma_{\varepsilon}\left(\theta_{\varepsilon}\right)$ and $\boldsymbol{a}(x, t, u, \nabla u)=\left|\nabla \beta_{\varepsilon}(u)\right|^{p-2} \nabla \beta_{\varepsilon}(u)$. We verify that the assumptions on page 16 of [6] are satisfied due to (3):

$$
\begin{aligned}
& \text { (A1) } \boldsymbol{a}(x, t, u, \nabla u) \cdot \nabla u=\left(\beta_{\varepsilon}^{\prime}(u)\right)^{p-1}|\nabla u|^{p} \geqslant\left(\frac{1}{1+\lambda L_{\varepsilon}}\right)^{p-1}|\nabla u|^{p} \\
& \text { (A2) }|\boldsymbol{a}(x, t, u, \nabla u)|=\left(\beta_{\varepsilon}^{\prime}(u)\right)^{p-1}|\nabla u|^{p-1} \leqslant|\nabla u|^{p-1} .
\end{aligned}
$$

We also see that (A1) is not satisfied uniformly since

$$
C_{0}(\varepsilon):=\left(\frac{1}{1+\lambda L_{\varepsilon}}\right)^{p-1} \rightarrow 0 \quad \text { as } \quad \varepsilon \rightarrow 0,
$$

which is in accordance with our expectations; in fact, that would imply the continuity of the enthalpy $\eta$ whereas this is only expected to hold for the temperature. 
We will show that there is a uniform, i.e. independent of $\varepsilon$, interior modulus of continuity for $\theta_{\varepsilon}$ and this will allow us to obtain a locally continuous temperature as a solution to the original problem as a consequence of the Theorem of Ascoli-Arzelá. The exact result is the following

THEOREM 1. - The sequence $\left(\theta_{\varepsilon}\right)_{\varepsilon}$ is locally equicontinuous, i.e., there is an independent of $\varepsilon$ interior modulus of continuity for $\theta_{\varepsilon}$. As a consequence, problem $(\mathbf{P})$ has at least a locally continuous solution.

We strongly emphasize that a uniqueness result for the problem is still missing, so we are actually only showing that the solutions obtained via this regularization procedure are locally continuous. Anyway, these are the interesting solutions in terms of the applications since the numerical methods use regularizations of this type.

The results presented here are local so we didn't have to associate to the equation a specific boundary value problem. We have just done it here to be consistent with the presentation of [11]. However, in section 7 we remark that this method can be extended up to the boundary in the case of Neumann or homogeneous Dirichlet conditions.

\section{3. - The local energy and logarithmic estimates.}

In this section we derive certain uniform local estimates that will be the main tool in the proof of the interior continuity.

Consider a cylinder $\left(x_{0}, t_{0}\right)+Q(\tau, \varrho) \subset Q$ and let $0 \leqslant \xi \leqslant 1$ be a piecewise smooth cutoff function in $\left(x_{0}, t_{0}\right)+Q(\tau, \varrho)$ such that

$$
|\nabla \zeta|<\infty \quad \text { and } \quad \zeta(x, t)=0, x \notin K_{Q}\left(x_{0}\right) .
$$

We start with the energy estimates. For the sake of simplicity and without loss of generality, we will state them for cylinders that are centered at the origin $(0,0)$, the changes being obvious in the case the center is a point $\left(x_{0}, t_{0}\right)$.

PROPOSITION 1. - Let $\theta_{\varepsilon}$ be a solution of $\left(P_{\varepsilon}\right)$ and $k<M$. There exists a constant $C>0$, that is independent of $\varepsilon$, such that for every cylinder $Q(\tau, \varrho) \subset Q$,

$$
\begin{aligned}
& \sup _{-\tau<t<0} \int_{K_{e} \times\{t\}}\left(\theta_{\varepsilon}-k\right)_{-}^{2} \xi^{p}+\int_{-\tau}^{0} \int_{K_{e}}\left|\nabla\left(\theta_{\varepsilon}-k\right)_{-} \zeta\right|^{p} \leqslant \\
& \leqslant C \int_{-\tau}^{0} \int_{K_{Q}}\left(\theta_{\varepsilon}-k\right)_{-}^{p}|\nabla \xi|^{p}+C \int_{K_{e} \times\{-\tau\}}\left(\theta_{\varepsilon}-k\right)_{-} \zeta^{p}+C \int_{-\tau}^{0} \int_{K_{e}}\left(\theta_{\varepsilon}-k\right)_{-} \xi^{p-1} \partial_{t} \zeta .
\end{aligned}
$$


Proof. - Let $\varphi=-\left(\theta_{\varepsilon}-k\right)_{-} \zeta^{p}$ in (5) and integrate in time over $(-\tau, t)$ for $t \in(-\tau, 0)$. The first term gives

$$
\begin{aligned}
&-\int_{-\tau}^{t} \int_{K_{Q}} \partial_{t}\left[\gamma_{\varepsilon}\left(\theta_{\varepsilon}\right)\right]\left(\left(\theta_{\varepsilon}-k\right)_{-} \xi^{p}\right)=\int_{K_{e}} \int_{-\tau}^{t} \partial_{t}\left(\int_{0}^{\left(\theta_{\varepsilon}-k\right)_{-}} \gamma_{\varepsilon}^{\prime}(k-s) s \mathrm{~d} s\right) \zeta^{p}= \\
& \quad=\int_{K_{e} \times\{t\}}\left(\int_{0}^{\left(\theta_{\varepsilon}-k\right)_{-}} \gamma_{\varepsilon}^{\prime}(k-s) s \mathrm{~d} s\right) \zeta^{p}-\int_{K_{e} \times\{-\tau\}}\left(\int_{0}^{\left(\theta_{\varepsilon}-k\right)_{-}} \gamma_{\varepsilon}^{\prime}(k-s) s \mathrm{~d} s\right) \zeta^{p}- \\
&-p \int_{-\tau}^{t} \int_{K_{e}}\left(\int_{0}^{\left(\theta_{\varepsilon}-k\right)_{-}} \gamma_{\varepsilon}^{\prime}(k-s) s \mathrm{~d} s\right) \zeta^{p-1} \partial_{t} \zeta \geqslant \\
& \geqslant \frac{1}{2} \int_{K_{Q} \times\{t\}}\left(\theta_{\varepsilon}-k\right)_{-}^{2} \zeta^{p}-2(M+\lambda) \int_{K_{e} \times\{-\tau\}}\left(\theta_{\varepsilon}-k\right)_{-} \zeta^{p}- \\
&-2 p(M+\lambda) \int_{-\tau}^{t} \int_{K_{e}}\left(\theta_{\varepsilon}-k\right)_{-} \zeta^{p-1} \partial_{t} \zeta,
\end{aligned}
$$

since we have, recalling (2),

$$
\int_{0}^{\left(\theta_{\varepsilon}-k\right)_{-}} \gamma_{\varepsilon}^{\prime}(k-s) s \mathrm{~d} s \geqslant \int_{0}^{\left(\theta_{\varepsilon}-k\right)_{-}} s \mathrm{~d} s=\frac{1}{2}\left(\theta_{\varepsilon}-k\right)_{-}^{2}
$$

and

$$
\begin{aligned}
\int_{0}^{\left(\theta_{\varepsilon}-k\right)_{-}} \gamma_{\varepsilon}^{\prime}(k-s) s d s & \leqslant\left(\theta_{\varepsilon}-k\right)_{-} \int_{0}^{\left(\theta_{\varepsilon}-k\right)_{-}} \gamma_{\varepsilon}^{\prime}(k-s) d s \\
& =\left(\theta_{\varepsilon}-k\right)_{-}\left[\gamma_{\varepsilon}(k)-\gamma_{\varepsilon}\left(\theta_{\varepsilon}\right)\right] \\
& \leqslant 2(M+\lambda)\left(\theta_{\varepsilon}-k\right)_{-} .
\end{aligned}
$$

Concerning the other term, we have

(9)

$$
\begin{aligned}
\int_{-\tau}^{t} \int_{K_{e}}\left|\nabla \theta_{\varepsilon}\right|^{p-2} \nabla \theta_{\varepsilon} \cdot \nabla\left[-\left(\theta_{\varepsilon}-k\right)_{-} \zeta^{p}\right]=\int_{-\tau}^{t} \int_{K_{e}}\left|\nabla\left(\theta_{\varepsilon}-k\right)_{-} \zeta\right|^{p}- \\
-p \int_{-\tau}^{t} \int_{K_{e}}\left|\nabla \theta_{\varepsilon}\right|^{p-2} \nabla \theta_{\varepsilon} \cdot \nabla \zeta\left[\zeta^{p-1}\left(\theta_{\varepsilon}-k\right)_{-}\right] \geqslant \\
\geqslant \frac{1}{2} \int_{-\tau}^{t} \int_{K_{e}}\left|\nabla\left(\theta_{\varepsilon}-k\right)_{-} \zeta\right|^{p}-C(p) \int_{-\tau}^{t} \int_{K_{e}}\left(\theta_{\varepsilon}-k\right)_{-}^{p}|\nabla \zeta|^{p},
\end{aligned}
$$


using the inequality of Young

$$
a b \leqslant \frac{\varepsilon^{p}}{p} a^{p}+\frac{1}{p^{\prime} \varepsilon^{p^{\prime}}} b^{p^{\prime}},
$$

valid for $a, b \geqslant 0$ and $\varepsilon>0$, with the choices

$$
a=\left(\theta_{\varepsilon}-k\right)_{-}|\nabla \xi|, \quad b=\left|\nabla\left(\theta_{\varepsilon}-k\right)_{-} \xi\right|^{p-1}
$$

and $\varepsilon$ appropriate. Since $t \in(-\tau, 0)$ is arbitrary, we can combine estimates (8) and (9) to obtain (7).

REMARK 1. - The price to be paid for the singularity at 0 , i.e., for the fact that $\gamma_{\varepsilon}^{\prime}$ is not uniformly bounded above in a neighbourhood of 0 , is the presence of $\left(\theta_{\varepsilon}-k\right)_{-}$ (with exponent 1) in the right hand side of (7), instead of $\left(\theta_{\varepsilon}-k\right)_{-}^{2}$ as in [6].

Proposition 2. - Let $\theta_{\varepsilon}$ be a solution of $\left(P_{\varepsilon}\right)$ and $k>\varepsilon$. There exists a constant $C>0$, that is independent of $\varepsilon$, such that for every cylinder $Q(\tau, \varrho) \subset Q$,

$$
\begin{aligned}
& \sup _{-\tau<t<0} \int_{K_{e} \times\{t\}}\left(\theta_{\varepsilon}-k\right)_{+}^{2} \xi^{p}+\int_{-\tau}^{0} \int_{K_{e}}\left|\nabla\left(\theta_{\varepsilon}-k\right)_{+} \xi\right|^{p} \leqslant \\
& \leqslant C \int_{-\tau}^{0} \int_{K_{Q}}\left(\theta_{\varepsilon}-k\right)_{+}^{p}|\nabla \xi|^{p}+C \int_{K_{Q} \times\{-\tau\}}\left(\theta_{\varepsilon}-k\right)_{+}^{2} \xi^{p}+C \int_{-\tau}^{0} \int_{K_{Q}}\left(\theta_{\varepsilon}-k\right)_{+}^{2} \xi^{p-1} \partial_{t} \zeta .
\end{aligned}
$$

Proof. - We are now above the singularity since we have $k>\varepsilon$. This means that $\gamma_{\varepsilon}^{\prime} \equiv 1$ and estimate (11) is exactly estimate (3.8) in [6] without the part coming from the absent lower order terms.

We proceed with the logarithmic estimates. Given constants $a, b, c$, with $0<c<a$, define the nonnegative function

$$
\begin{aligned}
\psi_{\{a, b, c\}}^{ \pm}(s) \equiv\left(\ln \left\{\frac{a}{(a+c)-(s-b)_{ \pm}}\right\}\right)_{+}= \\
= \begin{cases}\ln \left\{\frac{a}{(a+c) \pm(b-s)}\right\} & \text { if } b \pm c \lessgtr s \lessgtr b \pm(a+c) \\
0 & \text { if } s \leqq b \pm c\end{cases}
\end{aligned}
$$


whose first derivative is

$$
\left(\psi_{\{a, b, c\}}^{ \pm}\right)^{\prime}(s)= \begin{cases}\frac{1}{(b-s) \pm(a+c)} & \text { if } \mathrm{b} \pm \mathrm{c} \lessgtr \mathrm{s} \lessgtr \mathrm{b} \pm(\mathrm{a}+\mathrm{c}) \\ 0 & \text { if } s \lessgtr b \pm c\end{cases}
$$

and second derivative $\left(\psi_{\{a, b, c\}}^{ \pm}\right)^{\prime \prime}=\left\{\left(\psi_{\{a, b, c\}}^{+}\right)^{\prime}\right\}^{2} \geqslant 0$.

Now, given a bounded function $u$ in a cylinder $\left(x_{0}, t_{0}\right)+Q(\tau, \varrho)$ and a number $k$, define the constant

$$
H_{u, k}^{ \pm} \equiv \operatorname{ess}_{\left(x_{0}, t_{0}\right)+Q(\tau, \varrho)}\left|(u-k)_{ \pm}\right| .
$$

The following function was introduced in [4] and since then has been used as a recurrent tool in the proof of results concerning the local behaviour of solutions of degenerate PDE's:

$$
\Psi^{ \pm}\left(H_{u, k}^{ \pm},(u-k)_{ \pm}, c\right) \equiv \psi_{\left\{H_{u, k}^{ \pm}, k, c\right\}}^{ \pm} \circ u, \quad 0<c<H_{u, k}^{ \pm} .
$$

From now on, when referring to this function we will write $\psi^{ \pm}(u)$, omitting the subscripts; it will be clear what they are in every particular situation.

Take a cutoff function satisfying (6) as before and such that it is independent of $t \in(-\tau, 0)$. The logarithmic estimates, again in the case $\left(x_{0}, t_{0}\right)=(0,0)$, are

Proposition 3. - Let $\theta_{\varepsilon}$ be a solution of $\left(P_{\varepsilon}\right), k \in \mathbb{R}$ and $0<c<H_{\theta_{\varepsilon}}^{-}, k$. There exists a constant $C>0$, that is independent of $\varepsilon$, such that for every cylinder $Q(\tau, \varrho) \subset Q$,

$$
\begin{array}{r}
\sup _{-\tau<t<0} \int_{K_{Q} \times\{t\}}\left[\psi^{-}\left(\theta_{\varepsilon}\right)\right]^{2} \zeta^{p} \leqslant \int_{K_{Q} \times\{-\tau\}}\left(\int_{k}^{\theta_{\varepsilon}} \gamma_{\varepsilon}^{\prime}(s) \psi^{-}(s)\left(\psi^{-}\right)^{\prime}(s) \mathrm{d} s\right)_{+} \zeta^{p}+ \\
+C \int_{-\tau}^{0} \int_{K_{Q}} \psi^{-}\left(\theta_{\varepsilon}\right)\left|\left(\psi^{-}\right)^{\prime}\left(\theta_{\varepsilon}\right)\right|^{2-p}|\nabla \xi|^{p} .
\end{array}
$$

Proof. - Let $\varphi=2 \psi^{-}\left(\theta_{\varepsilon}\right)\left[\left(\psi^{-}\right)^{\prime}\left(\theta_{\varepsilon}\right)\right] \zeta^{p}$ in (5) and integrate in time over $(-\tau, t)$ for $t \in(-\tau, 0)$. We have, recalling that $\partial_{t} \zeta \equiv 0$,

$$
\begin{aligned}
& \int_{-\tau}^{t} \int_{K_{Q}} \partial_{t}\left[\gamma_{\varepsilon}\left(\theta_{\varepsilon}\right)\right]\left(2 \psi^{-}\left(\theta_{\varepsilon}\right)\left[\left(\psi^{-}\right)^{\prime}\left(\theta_{\varepsilon}\right)\right] \zeta^{p}\right)= \\
& \quad=\int_{-\tau}^{t} \int_{K_{Q}} \partial_{t}\left\{\left(\int_{k}^{\theta_{\varepsilon}} 2 \gamma_{\varepsilon}^{\prime}(s) \psi^{-}(s)\left(\psi^{-}\right)^{\prime}(s) \mathrm{d} s\right)\right\} \zeta^{p} \geqslant \\
& \geqslant \int_{K_{Q} \times\{t\}}\left[\psi^{-}\left(\theta_{\varepsilon}\right)\right]^{2} \zeta^{p}-\int_{K_{e} \times\{-\tau\}}\left(\int_{k}^{\theta_{\varepsilon}} 2 \gamma_{\varepsilon}^{\prime}(s) \psi^{-}(s)\left(\psi^{-}\right)^{\prime}(s) \mathrm{d} s\right)_{+}, \xi^{p},
\end{aligned}
$$


due to the fact that, using (2),

$$
\begin{aligned}
\left(\int_{k}^{\theta_{\varepsilon}} 2 \gamma_{\varepsilon}^{\prime}(s) \psi^{-}(s)\left(\psi^{-}\right)^{\prime}(s) \mathrm{d} s\right)_{+} \geqslant \int_{k}^{\theta_{\varepsilon}} 2 \psi^{-}(s)\left(\psi^{-}\right)^{\prime}(s) \mathrm{d} s= \\
=\left[\psi^{-}\left(\theta_{\varepsilon}\right)\right]^{2}-\left(\psi^{-}(k)\right)^{2}=\left[\psi^{-}\left(\theta_{\varepsilon}\right)\right]^{2}
\end{aligned}
$$

because $\psi^{-}(k)=0$. The other term gives

$$
\begin{aligned}
& \int_{-\tau}^{t} \int_{K_{Q}}\left|\nabla \theta_{\varepsilon}\right|^{p-2} \nabla \theta_{\varepsilon} \cdot \nabla\left\{2 \psi^{-}\left(\theta_{\varepsilon}\right)\left[\left(\psi^{-}\right)^{\prime}\left(\theta_{\varepsilon}\right)\right] \zeta^{p}\right\}= \\
& =\int_{-\tau}^{t} \int_{K_{q}}\left|\nabla \theta_{\varepsilon}\right|^{p}\left\{2\left(1+\psi^{-}\left(\theta_{\varepsilon}\right)\right)\left[\left(\psi^{-}\right)^{\prime}\left(\theta_{\varepsilon}\right)\right]^{2} \zeta^{p}\right\}+ \\
& +p \int_{-\tau}^{t} \int_{K_{Q}}\left|\nabla \theta_{\varepsilon}\right|^{p-2} \nabla \theta_{\varepsilon} \cdot \nabla \xi\left\{2 \psi^{-}\left(\theta_{\varepsilon}\right)\left[\left(\psi^{-}\right)^{\prime}\left(\theta_{\varepsilon}\right)\right] \zeta^{p-1}\right\} \geqslant \\
& \geqslant \int_{-\tau}^{t} \int_{K_{Q}}\left|\nabla \theta_{\varepsilon}\right|^{p}\left\{2\left(1+\psi^{-}\left(\theta_{\varepsilon}\right)-\psi^{-}\left(\theta_{\varepsilon}\right)\right)\left[\left(\psi^{-}\right)^{\prime}\left(\theta_{\varepsilon}\right)\right]^{2} \zeta^{p}\right\}- \\
& -2(p-1)^{p-1} \int_{-\tau}^{t} \int_{K_{Q}} \psi^{-}\left(\theta_{\varepsilon}\right)\left|\left(\psi^{-}\right)^{\prime}\left(\theta_{\varepsilon}\right)\right|^{2-p}|\nabla \zeta|^{p} \geqslant \\
& \geqslant-C \int_{-\tau}^{t} \int_{K_{e}} \psi^{-}\left(\theta_{\varepsilon}\right)\left|\left(\psi^{-}\right)^{\prime}\left(\theta_{\varepsilon}\right)\right|^{2-p}|\nabla \xi|^{p},
\end{aligned}
$$

using again Young's inequality (10) with $\varepsilon=(p-1)^{(p-1) / p}$ and

$$
a=\left|\left(\psi^{-}\right)^{\prime}\left(\theta_{\varepsilon}\right)\right|^{2 / p-1}|\nabla \zeta|, \quad b=\left|\nabla \theta_{\varepsilon}\right|^{p-1} \xi^{p-1}\left|\left(\psi^{-}\right)^{\prime}\left(\theta_{\varepsilon}\right)\right|^{2-2 / p} .
$$

Since $t \in(-\tau, 0)$ is arbitrary, we can combine both estimates to obtain (12).

REMARK 2. - In this estimate there is a term that depends on $\varepsilon$ through $\gamma_{\varepsilon}^{\prime}$. We will see later how to avoid this difficulty.

The proof of the next proposition follows from the same remark that was used in the proof of Proposition 2. 
Proposition 4. - Let $\theta_{\varepsilon}$ be a solution of $\left(P_{\varepsilon}\right), k>\varepsilon$ and $0<c<H_{\theta_{\varepsilon}}^{+}, k$. There exists a constant $C>0$, that is independent of $\varepsilon$, such that for every cylinder $Q(\tau, \varrho) \subset Q$,

$$
\begin{aligned}
\sup _{-\tau<t<0} \int_{K_{e} \times\{t\}}\left[\psi^{+}\left(\theta_{\varepsilon}\right)\right]^{2} \zeta^{p} \leqslant \int_{K_{e} \times\{-\tau\}}\left[\psi^{+}\left(\theta_{\varepsilon}\right)\right]^{2} \zeta^{p}+ \\
+C \int_{-\tau}^{0} \int_{K_{e}} \psi^{+}\left(\theta_{\varepsilon}\right)\left|\left(\psi^{+}\right)^{\prime}\left(\theta_{\varepsilon}\right)\right|^{2-p}|\nabla \zeta|^{p} .
\end{aligned}
$$

\section{4. - The geometric setting and the alternative.}

The proof of the equicontinuity will follow from these estimates, adapting the technique introduced by DiDenedetto (cf. [6]). It consists essentially in showing that for every point $\left(x_{0}, t_{0}\right) \in Q$ we can find a sequence of nested and shrinking cylinders $\left(x_{0}, t_{0}\right)+Q\left(\tau_{n}, \varrho_{n}\right)$, such that the essential oscillation of each function $\theta_{\varepsilon}$ in these cylinders goes to zero as $n \rightarrow \infty$ in a way that is qualitatively independent of $\varepsilon$.

This can be achieved, roughly speaking, by considering the equation in a geometry dictated by its own structure. This means that, instead of the usual cylinders, we have to work in cylinders whose dimensions take the degeneracy of the equation into account. Let's make this idea precise. From now on we will drop the $\varepsilon$ in $\theta_{\varepsilon}$.

Consider a point $\left(x_{0}, t_{0}\right) \in Q$ and, by translation and to simplify, assume $\left(x_{0}, t_{0}\right)=$ $=(0,0)$. Consider $R>0$ such that $Q\left(R^{p-1}, 2 R\right) \subset Q$, define

$$
\mu_{-}:=\underset{Q\left(R^{p-1}, 2 R\right)}{\operatorname{essinf}} \theta ; \quad \mu_{+}:=\underset{Q\left(R^{p-1}, 2 R\right)}{\operatorname{ess} \sup } \theta ; \quad \omega:=\underset{Q\left(R^{p-1}, 2 R\right)}{\operatorname{ess} \text { osc }} \theta=\mu_{+}-\mu_{-}
$$

and construct the cylinder

$$
Q\left(a_{0} R^{p}, R\right), \quad \text { with } a_{0}=\left(\frac{\omega}{A}\right)^{2-p}
$$

where the number $A$ will be chosen in the course of the proof of the form

$$
A=2^{s_{3}}, \quad \text { with } \quad s_{3}>C \omega^{-\alpha}, \quad \alpha=\frac{2(p+1)(N+p)}{p} .
$$

Note that for $p=2$, i.e. in the nondegenerate case, $a_{0}=1$ and these are the standard parabolic cylinders. We will assume, without loss of generality, that $\omega<1$ and also that

$$
\frac{1}{a_{0}}=\left(\frac{\omega}{A}\right)^{p-2}>R
$$


and comment later on the case when this doesn't hold. Now, (15) implies that $Q\left(a_{0} R^{p}, R\right) \subset Q\left(R^{p-1}, 2 R\right)$ and the relation

$$
\underset{Q\left(a_{0} R^{P}, R\right)}{\operatorname{ess} \operatorname{osc}} \theta \leqslant \omega
$$

which will be the starting point of an iteration process that leads to our main results. Note that we had to consider the cylinder $Q\left(R^{p-1}, 2 R\right)$ and assume (15), so that (16) would hold for the rescalled cylinder $Q\left(a_{0} R^{p}, R\right)$. This is in general not true for a given cylinder since its dimensions would have to be intrinsically defined in terms of the essential oscillation of the function within it. We now consider subcylinders of $Q\left(a_{0} R^{p}, R\right)$ of the form

$$
\left(0, t^{*}\right)+Q\left(d R^{p}, R\right), \quad \text { with } d=\left(\frac{\omega}{2}\right)^{2-p}
$$

that are contained in $Q\left(a_{0} R^{p}, R\right)$, since $A>2$ and if

$$
\left(2^{p-2}-A^{p-2}\right) \frac{R^{p}}{\omega^{p-2}}<t^{*}<0
$$

The proof of our main result (Theorem 2 ahead) follows from the analysis of two complementary cases and the achievement of the same type of conclusion for both. We can briefly describe them in the following way: in the first case we assume that there is a cylinder of the type $\left(0, t^{*}\right)+Q\left(d R^{p}, R\right)$ where $\theta$ is essentially away from its infimum. We show that going down to a smaller cylinder the oscillation decreases by a small factor that we can exhibit and that depends on the oscillation. If that cylinder can not be found then $\theta$ is essentially away from its supremum in all cylinders of that type and we can add up this information to reach the same conclusion as in the previous case. We state this in a precise way.

For a constant $\nu_{0} \in(0,1)$, that will be determined depending only on the data and $\omega$, we will assume that either

(C1) There is a cylinder of the type $\left(0, t^{*}\right)+Q\left(d R^{p}, R\right)$ for which

$$
\frac{\left|\left\{(x, t) \in\left(0, t^{*}\right)+Q\left(d R^{p}, R\right): \theta(x, t)<\mu_{-}+\frac{\omega}{2}\right\}\right|}{\left|Q\left(d R^{p}, R\right)\right|} \leqslant v_{0}
$$


or this does not hold. In that case we have the complementary condition, and since $\mu_{+}-(\omega / 2) \geqslant \mu_{-}+(\omega / 2)$, it can be stated as

(C2) For every cylinder of the type $\left(0, t^{*}\right)+Q\left(d R^{p}, R\right)$

$$
\frac{\left|\left\{(x, t) \in\left(0, t^{*}\right)+Q\left(d R^{p}, R\right): \theta(x, t)>\mu_{+}-\frac{\omega}{2}\right\}\right|}{\left|Q\left(d R^{p}, R\right)\right|} \leqslant 1-v_{0}
$$

\section{5. - Analysis of case (C1).}

Lemma 1. - There exists a constant $v_{0} \in(0,1)$, depending only on the data and $\omega$, such that if (17) holds then

$$
\theta(x, t)>\mu_{-}+\frac{\omega}{4} \quad \text { a.e. }(x, t) \in\left(0, t^{*}\right)+Q\left(d\left(\frac{R}{2}\right)^{p}, \frac{R}{2}\right)
$$

Proof. - Take the cylinder for which (17) holds and assume, by translation, that $t^{*}=0$. Let

$$
R_{n}=\frac{R}{2}+\frac{R}{2^{n+1}}, \quad n=0,1, \ldots,
$$

and construct the family of nested and shrinking cylinders $Q\left(d R_{n}^{p}, R_{n}\right)$. Consider piecewise smooth cutoff functions $0<\zeta_{n} \leqslant 1$, defined in these cylinders, and satisfying the following set of assumptions

$$
\begin{array}{ccc}
\zeta_{n}=1 \quad \text { in } Q\left(d R_{n+1}^{p}, R_{n+1}\right) & \zeta_{n}=0 \quad \text { on } \partial_{p} Q\left(d R_{n}^{p}, R_{n}\right) \\
\left|\nabla \zeta_{n}\right| \leqslant \frac{2^{n+1}}{R} & 0 \leqslant \partial_{t} \zeta_{n} \leqslant \frac{2^{p(n+1)}}{d R^{p}} .
\end{array}
$$

Write the energy inequality (7) for the functions $\left(\theta-k_{n}\right)_{-}$, with

$$
k_{n}=\mu_{-}+\frac{\omega}{4}+\frac{\omega}{2^{n+2}}, \quad n=0,1, \ldots
$$


in the cylinders $Q\left(d R_{n}^{p}, R_{n}\right)$ and with $\zeta=\xi_{n}$. They read

$$
\begin{aligned}
& \sup _{-d R_{n}^{p}<t<0} \int_{K_{R_{n}} \times\{t\}}\left(\theta-k_{n}\right)_{-}^{2} \zeta_{n}^{p}+\int_{-d R_{n}^{p}}^{0} \int_{K_{R_{n}}}\left|\nabla\left(\theta-k_{n}\right)_{-} \zeta_{n}\right|^{p} \leqslant \\
& \leqslant C \int_{-d R_{n}^{p}}^{0} \int_{K_{R_{n}}}\left(\theta-k_{n}\right)_{-}^{p}\left|\nabla \zeta_{n}\right|^{p}+C \int_{-d R_{n}^{p}}^{0} \int_{K_{R_{n}}}\left(\theta-k_{n}\right)_{-} \zeta_{n}^{p-1} \partial_{t} \zeta_{n} \leqslant \\
& \leqslant C \frac{2^{p(n+1)}}{R^{p}}\left\{\int_{-d R_{n}^{p}}^{0} \int_{K_{R_{n}}}\left(\theta-k_{n}\right)_{-}^{p}+\frac{1}{d} \int_{-d R_{n}^{p}}^{0} \int_{K_{R_{n}}}\left(\theta-k_{n}\right)_{-}\right\} .
\end{aligned}
$$

Next, observing that, when it is not zero,

$$
\left(\theta-k_{n}\right)_{-}=\left(\mu_{-}-\theta\right)+\frac{\omega}{4}+\frac{\omega}{2^{n+2}} \leqslant \frac{\omega}{2}
$$

so that, since $2-p<0$,

$$
\left(\theta-k_{n}\right)_{-}^{2}=\left(\theta-k_{n}\right)_{-}^{2-p}\left(\theta-k_{n}\right)_{-}^{p} \geqslant\left(\frac{\omega}{2}\right)^{2-p}\left(\theta-k_{n}\right)_{-}^{p},
$$

we obtain from (19)

$$
\begin{aligned}
\left(\frac{\omega}{2}\right)^{2-p} \sup _{-d R_{n}^{p}<t<0} \int_{K_{R_{n}} \times\{t\}}\left(\theta-k_{n}\right)_{-}^{p} \xi_{n}^{p}+\int_{-d R_{n}^{p}}^{0} \int_{K_{R_{n}}}\left|\nabla\left(\theta-k_{n}\right)_{-} \zeta_{n}\right|^{p} \leqslant \\
\leqslant C \frac{2^{p(n+1)}}{R^{p}}\left\{\left(\frac{\omega}{2}\right)^{p}+\frac{1}{d}\left(\frac{\omega}{2}\right)\right\} \int_{-d R_{n}^{p}}^{0} \int_{K_{R_{n}}}^{0} \chi_{\left\{\left(\theta-k_{n}\right)_{-}>0\right\}} .
\end{aligned}
$$

Recall that $d=(\omega / 2)^{2-p}$ and divide (20) by $d$ to get

$$
\begin{aligned}
& \sup _{-d R_{n}^{p}<t<0} \int_{K_{R_{n}} \times\{t\}}\left(\theta-k_{n}\right)_{-}^{p} \zeta_{n}^{p}+\frac{1}{d} \int_{-d R_{n}^{p}}^{0} \int_{K_{R_{n}}}\left|\nabla\left(\theta-k_{n}\right)_{-} \zeta_{n}\right|^{p} \leqslant \\
& \leqslant C \frac{2^{p(n+1)}}{R^{p}}\left(\frac{\omega}{2}\right)^{p}\left(1+\frac{2}{\omega}\right) \frac{1}{d} \int_{-d R_{n}^{p} K_{R_{n}}^{0} \chi_{\left\{\left(\theta-k_{n}\right)_{-}>0\right\}} \leqslant} \leqslant \frac{C}{\omega} \frac{2^{p}}{R^{p}}\left(\frac{\omega}{2}\right)^{p} \frac{1}{d} \int_{-d R_{n}^{p}}^{0} \int_{K_{R_{n}}} \chi_{\left\{\left(\theta-k_{n}\right)_{-}>0\right\}},
\end{aligned}
$$


because $\omega \leqslant 1$. Now we perform a change of the time variable in (21), putting $\bar{t}=t / d$ and defining

$$
\bar{\theta}(\cdot, \bar{t})=\theta(\cdot, t) \quad \text { and } \quad \bar{\zeta}_{n}(\cdot, \bar{t})=\zeta_{n}(\cdot, t),
$$

and obtain the simplified inequality

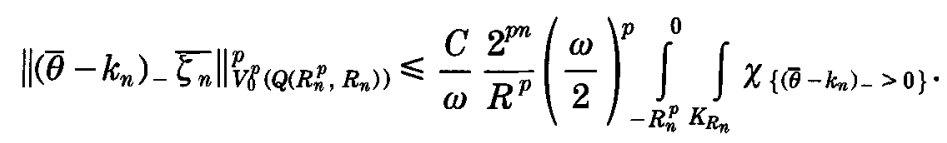

Define, for each $n$,

$$
A_{n}=\int_{-R_{n}^{p}}^{0} \int_{R_{R_{n}}} \chi_{\left.\left\{\bar{\theta}-k_{n}\right)_{-}>0\right\}} \mathrm{d} x \mathrm{~d} \bar{t}
$$

and observe that the following estimates hold

$$
\frac{1}{2^{p(n+2)}}\left(\frac{\omega}{2}\right)^{p} A_{n+1} \leqslant\left|k_{n}-k_{n+1}\right|^{p} A_{n+1} \leqslant\left\|\left(\bar{\theta}-k_{n}\right)_{-}\right\|_{p, Q\left(R_{n+1}^{p}, R_{n+1}\right)}^{p} \leqslant
$$

$\leqslant\left\|\left(\bar{\theta}-k_{n}\right)_{-} \bar{\zeta}_{n}\right\|_{p, Q\left(R_{n}^{p}, R_{n}\right)}^{p} \leqslant C\left\|\left(\bar{\theta}-k_{n}\right)_{-} \bar{\zeta}_{n}\right\|_{V_{0}^{p}\left(Q\left(R_{n}^{p}, R_{n}\right)\right)}^{p} A_{n}^{p /(N+p)} \leqslant \frac{C}{\omega} \frac{2^{p n}}{R^{p}}\left(\frac{\omega}{2}\right)^{p} A_{n}^{1+(p /(N+p))}$.

In fact, the first and the third inequalities are obvious; the second one holds due to the fact that $k_{n+1}<k_{n}$; the fourth inequality is a consequence of a well known imbedding theorem (see, e.g., Corollary 3.1 on chapter I of [6]) and the last one follows from (22). Next, define the numbers

$$
X_{n}=\frac{A_{n}}{\left|Q\left(R_{n}^{p}, R_{n}\right)\right|},
$$

divide (23) by $\left|Q\left(R_{n+1}^{p}, R_{n+1}\right)\right|$ and obtain the recursive relation

$$
X_{n+1} \leqslant \frac{C 4^{p n}}{\omega} X_{n}^{1+(p /(N+p))}
$$

since

$$
\begin{aligned}
\frac{\left|Q\left(R_{n}^{p}, R_{n}\right)\right|^{1+\frac{p}{N+p}}}{\left|Q\left(R_{n+1}^{p}, R_{n+1}\right)\right| R^{p}}=\frac{\left(\left(2 R_{n}\right)^{N+p}\right)^{1+(p /(N+p))}}{\left(2 R_{n+1}\right)^{N+p} R^{p}} & =\frac{\left(\left(2^{n}+1 / 2^{n}\right) R\right)^{N+2 p}}{\left(\left(2^{n}+2 / 2^{n+1}\right) R\right)^{N+p} R^{p}}= \\
& =2^{N+p}\left(\frac{2^{n}+1}{2^{n}+2}\right)^{N+p}\left(\frac{2^{n}+1}{2^{n}}\right)^{p} \leqslant 2^{N+2 p} .
\end{aligned}
$$


We can use a lemma on the fast geometric convergence of sequences (see Lemma 4.1 on Chapter I of [6]) to conclude that if

$$
X_{0} \leqslant\left(\frac{\omega}{C}\right)^{(N+p) / p} 4^{-(N+p)^{2} / p}=C \omega^{(N+p) / p} \equiv \nu_{0}
$$

then

$$
X_{n} \rightarrow 0 \text {. }
$$

But (24) is nothing but the assumption (17) of the lemma and the conclusion easily follows from (25). In fact, observe that

$$
R_{n} \searrow \frac{R}{2} \quad \text { and } \quad k_{n} \searrow \mu_{-}+\frac{\omega}{4},
$$

and since (25) implies that $A_{n} \rightarrow 0$, we conclude that

$$
\begin{aligned}
\left|\left\{(x, \bar{t}) \in Q\left(\left(\frac{R}{2}\right)^{p}, \frac{R}{2}\right): \bar{\theta}(x, \bar{t}) \leqslant \mu_{-}+\frac{\omega}{4}\right\}\right|= \\
=\left|\left\{(x, t) \in Q\left(d\left(\frac{R}{2}\right)^{p}, \frac{R}{2}\right): \theta(x, t) \leqslant \mu_{-}+\frac{\omega}{4}\right\}\right|=0
\end{aligned}
$$

and the lemma is proved.

Our next aim is to show that the conclusion of Lemma 1 holds in a full cylinder $Q(\tau, \varrho)$. The idea is to use the fact that at the time level

$$
-\hat{t}:=t^{*}-d\left(\frac{R}{2}\right)^{p}
$$

the function $\theta(x)$ is strictly above the level $\mu_{-}+\omega / 4$ in the cube $K_{R / 2}$ and look at this time level as an initial condition to make the conclusion hold up to $t=0$. Again this is a sophisticated way of showing that the equation behaves like the heat equation. As an intermediate step we need the following lemma.

LEMMA 2. - Given $\nu_{1} \in(0,1)$, there exists $s_{1} \in \mathbb{N}$, depending on the data and $\omega$, such that

$$
\left|\left\{x \in K_{R / 4}: \theta(x, t)<\mu_{-}+\frac{\omega}{2^{s_{1}}}\right\}\right| \leqslant v_{1}\left|K_{R / 4}\right|, \quad \forall t \in(-\hat{t}, 0) .
$$


Proof. - We use the logarithmic estimate (12) applied to the function $(\theta-k)_{-}$in the cylinder $Q(\tilde{t}, R / 2)$, with the choices

$$
k=\mu_{-}+\frac{\omega}{4} \quad \text { and } \quad c=\frac{\omega}{2^{n+2}}
$$

where $n \in \mathbb{N}$ will be chosen later. We have

$$
k-\theta \leqslant H_{\theta, k}^{-}=\underset{Q(i, R / 2)}{\operatorname{ess} \sup _{i}}\left|\left(\theta-\mu_{-}-\frac{\omega}{4}\right)_{-}\right| \leqslant \frac{\omega}{4}
$$

and, since if $H_{\theta, k}^{-}=0$ the result is trivial, we may assume it is strictly positive and choose $n$ big enough so that $c<H_{\theta, k}^{-}$. We recall that in this case the logarithmic function is defined in the whole domain of $\theta, Q(\hat{t}, R / 2)$ (since it is obvious that $H_{\theta, k}+\theta-$ $-k+c>0$ ), and given by

$$
\Psi^{-}=\psi_{\left\{H_{\bar{\theta}, k}, k, \omega / 2^{n+2}\right\}}(\theta)= \begin{cases}\ln \left\{\frac{H_{\theta, k}^{-}}{\left.H_{\theta, k}^{-}+\theta-k+\omega / 2^{n+2}\right\}}\right. & \text { if } \theta<k-\frac{\omega}{2^{n+2}} \\ 0 & \text { if } \theta \geqslant k-\frac{\omega}{2^{n+2}} .\end{cases}
$$

From (27), we can easily estimate

$$
\Psi^{-} \leqslant n \ln 2 \quad \text { since } \quad \frac{H_{\theta, k}^{-}}{H_{\theta, k}^{-}+\theta-k+\omega / 2^{n+2}} \leqslant \frac{\omega / 4}{\omega / 2^{n+2}}=2^{n}
$$

and the derivative (here in the nonvanishing case $\theta<k-c$ )

$$
\begin{aligned}
&\left|\left(\psi^{-}\right)^{\prime}(\theta)\right|^{2-p}=\left|\frac{-1}{H_{\theta, k}^{-}+\theta-k+c}\right|^{2-p}=\left(H_{\theta, k}^{-}+\theta-\right.k+c)^{p-2} \leqslant \\
& \qquad \leqslant\left(\frac{\omega}{4}\right)^{p-2} \leqslant\left(\frac{\omega}{2}\right)^{p-2} .
\end{aligned}
$$

Now observe that as a consequence of Lemma 1 , we have $\theta(x,-\hat{t})>k$ in the cube $K_{R / 2}$, which implies that

$$
\Psi^{-}(x,-\hat{t})=0, x \in K_{R / 2} .
$$

Choosing a piecewise smooth cutoff function $0<\zeta(x) \leqslant 1$, defined on $K_{R / 2}$ and such that

$$
\zeta=1 \text { in } K_{R / 4} \quad \text { and } \quad|\nabla \zeta| \leqslant \frac{8}{R}
$$


inequality (12) reads

$$
\sup _{-\hat{t}<t<0} \int_{K_{R / 2} \times\{t\}}\left[\psi^{-}(\theta)\right]^{2} \zeta^{p} \leqslant C \int_{-\hat{t}}^{0} \int_{K_{R / 2}} \psi^{-}(\theta)\left|\left(\psi^{-}\right)^{\prime}(\theta)\right|^{2-p}|\nabla \zeta|^{p} .
$$

The right hand side is estimated above, using (28) and (29), by

$C n(\ln 2)\left(\frac{\omega}{2}\right)^{p-2}\left(\frac{8}{R}\right)^{p} \hat{t}\left|K_{R / 2}\right| \leqslant C n\left(\frac{\omega}{2}\right)^{p-2}\left(\frac{8}{R}\right)^{p}\left(\frac{\omega}{A}\right)^{2-p} R^{p} 2^{N}\left|K_{R / 4}\right| \leqslant C n A^{p-2}\left|K_{R / 4}\right|$ observing also that

$$
\hat{t} \leqslant a_{0} R^{p}=\left(\frac{\omega}{A}\right)^{2-p} R^{p} .
$$

Since the integrand is nonnegative, we estimate the left hand side of (30) below integrating over the smaller set

$$
S=\left\{x \in K_{R / 4}: \theta(x, t)<\mu_{-}+\frac{\omega}{2^{n+2}}\right\} \subset K_{R / 2}
$$

and observing that in $S, \zeta=1$ and

$$
\frac{H_{\theta, k}^{-}}{H_{\theta, k}^{-}+\theta-k+\frac{\omega}{2^{n+2}}} \geqslant \frac{\left(H_{\theta, k}^{-}-\omega / 4\right)+\omega / 4}{\left(H_{\theta, k}^{-}-\omega / 4\right)+\omega / 2^{n+1}} \geqslant \frac{\omega / 4}{\omega / 2^{n+1}}=2^{n-1},
$$

because $\left(H_{\theta, k}^{-}-\omega / 4\right) \leqslant 0$ and $(\omega / 4)>\left(\omega / 2^{n+1}\right), n>1$, which implies that

$$
\left[\psi^{-}(\theta)\right]^{2} \geqslant\left[\ln \left(2^{n-1}\right)\right]^{2}=(n-1)^{2}(\ln 2)^{2} .
$$

From both estimates we get $(n-1)^{2}(\ln 2)^{2}|S| \leqslant C n A^{p-2}\left|K_{R / 4}\right|$, i.e.

$$
\left|\left\{x \in K_{R / 4}: \theta(x, t)<\mu_{-}+\frac{\omega}{2^{n+2}}\right\}\right| \leqslant C \frac{n}{(n-1)^{2}} A^{p-2}\left|K_{R / 4}\right|
$$

and to prove the lemma we just need to choose

$$
s_{1}=n+2 \quad \text { with } \quad n>1+\frac{2 C}{v_{1}} A^{p-2},
$$

since if $n \geqslant 1+2 / \alpha$ then $n /(n-1)^{2} \leqslant \alpha, \alpha>0$

REMARK 3. - A crucial step towards the proof of the equicontinuity occured in the previous proof. In fact, this is the only place where we make use of estimate (12), that 
has a term depending on $\varepsilon$, namely

$$
\int_{K_{e} \times\{-\tau\}}\left(\int_{k}^{\theta_{\varepsilon}} \gamma_{\varepsilon}^{\prime}(s) \psi^{-}(s)\left(\psi^{-}\right)^{\prime}(s) d s\right)_{+} \xi^{p} .
$$

We get rid of it (and keep the independence of $\varepsilon$ ) by choosing appropriately the initial time $-\tau$ for which $\Psi^{-}$vanishes.

We now state the main result of this section.

Proposition 5. - There exist constants $\nu_{0} \in(0,1), 1<s_{1} \in \mathbb{N}$, depending on the data and $\omega$, such that if (17) holds then

$$
\theta(x, t)>\mu_{-}+\frac{\omega}{2^{s_{1}+1}} \quad \text { a.e. } \quad(x, t) \in Q\left(\hat{t}, \frac{R}{8}\right) .
$$

Proof. - Consider the cylinder for which (17) holds, let

$$
R_{n}=\frac{R}{8}+\frac{R}{2^{n+3}}, \quad n=0,1, \ldots
$$

and construct the family of nested and shrinking cylinders $Q\left(\hat{t}, R_{n}\right)$, where $\hat{t}$ is given by (26). Take piecewise smooth cutoff functions $0<\zeta_{n}(x) \leqslant 1$, not depending on $t$, defined in $K_{R_{n}}$ and satisfying the assumptions

$$
\zeta_{n}=1 \text { in } K_{R_{n+1}} \quad\left|\nabla \zeta_{n}\right| \leqslant \frac{2^{n+4}}{R} .
$$

Write the local energy inequalities (7) for the functions $\left(\theta-k_{n}\right)_{-}$in the cylinders $Q\left(\hat{t}, R_{n}\right)$, with

$$
k_{n}=\mu_{-}+\frac{\omega}{2^{s_{1}+1}}+\frac{\omega}{2^{s_{1}+1+n}}, \quad n=0,1, \ldots,
$$

$\left(s_{1}>1\right.$ is to be chosen) and $\zeta=\zeta_{n}$. Observing that, due to Lemma 1 , we have $\theta(x,-\hat{t})>$ $>\mu_{-}+(\omega / 4) \geqslant k_{n}$ in the cube $K_{R / 2} \supset K_{R_{n}}$, which implies that

$$
\left(\theta-k_{n}\right)_{-}(x,-\hat{t})=0, x \in K_{R_{n}}, \quad n=0,1, \ldots,
$$

they read

$$
\begin{aligned}
& \sup _{-\hat{t}<t<0} \int_{K_{R_{n}} \times\{t\}}\left(\theta-k_{n}\right)_{-}^{2} \xi_{n}^{p}+\int_{-\hat{t}}^{0} \int_{K_{R_{n}}}\left|\nabla\left(\theta-k_{n}\right)_{-} \xi_{n}\right|^{p} \leqslant \\
& \leqslant C \int_{-\hat{t}}^{0} \int_{K_{R_{n}}}\left(\theta-k_{n}\right)_{-}^{p}\left|\nabla \xi_{n}\right|^{p} \leqslant C \frac{2^{p(n+4)}}{R^{p}} \int_{-\hat{t}}^{0} \int_{K_{R_{n}}}^{0}\left(\theta-k_{n}\right)_{-}^{p} .
\end{aligned}
$$


Next, since $\left(\theta-k_{n}\right)_{-} \leqslant\left(\omega / 2^{s_{1}}\right)$ and $2-p<0$, we have, using also (31),

$$
\begin{aligned}
\left(\theta-k_{n}\right)_{-}^{2} \geqslant\left(\frac{\omega}{2^{s_{1}}}\right)^{2-p}\left(\theta-k_{n}\right)_{-}^{p} & \geqslant 2^{-p}\left(\frac{2^{s_{1}}}{A}\right)^{p-2} \frac{\hat{t}}{(R / 2)^{p}}\left(\theta-k_{n}\right)_{-}^{p} \\
& \geqslant \frac{\hat{t}}{(R / 2)^{p}}\left(\theta-k_{n}\right)_{-}^{p},
\end{aligned}
$$

choosing $s_{1}>\log _{2} A+p /(p-2)$. So, from (33), dividing by $\hat{t} /(R / 2)^{p}$, we get

$$
\begin{aligned}
\sup _{-\hat{t}<t<0} \int_{K_{R_{n}} \times\{t\}}\left(\theta-k_{n}\right)_{-}^{p} \xi_{n}^{p}+\frac{(R / 2)^{p}}{\hat{t}} \int_{-\hat{t}}^{0} \int_{K_{R_{n}}}\left|\nabla\left(\theta-k_{n}\right)_{-} \xi_{n}\right|^{p} & \leqslant \\
& \leqslant C \frac{2^{p(n+3)}}{\hat{t}} \int_{-\hat{t}}^{0} \int_{K_{R_{n}}}^{0}\left(\theta-k_{n}\right)_{-}^{p} .
\end{aligned}
$$

The change of the time variable $\bar{t}=t\left((R / 2)^{p} / \hat{t}\right)$, with the new function

$$
\bar{\theta}(\cdot, \bar{t})=\theta(\cdot, t),
$$

leads to the simplified inequality

$$
\left\|\left(\bar{\theta}-k_{n}\right)-\zeta_{n}\right\|_{V^{p}\left(Q\left((R / 2)^{p}, R_{n}\right)\right)}^{p} \leqslant C \frac{2^{p m}}{(R / 2)^{p}}\left(\frac{\omega}{2^{s_{1}}}\right)_{-(R / 2)^{p}}^{p} \int_{K_{R_{n}}}^{0} \chi_{\left\{\left(\bar{\theta}-k_{n}\right)_{-}>0\right\}} .
$$

Define, for each $n$,

$$
A_{n}=\int_{-(R / 2)^{p}}^{0} \int_{K_{R_{n}}} \chi_{\left\{\left(\bar{\theta}-k_{n}\right)_{-}>0\right\}} \mathrm{d} x \mathrm{~d} \bar{t}
$$

and observe that the following estimates hold, by a reasoning similar to the one that led to (23):

$$
\begin{gathered}
\frac{1}{2^{p(n+2)}}\left(\frac{\omega}{2^{s_{1}}}\right)^{p} A_{n+1} \leqslant\left|k_{n}-k_{n+1}\right|^{p} A_{n+1} \leqslant\left\|\left(\bar{\theta}-k_{n}\right)_{-}\right\|_{p, Q\left((R / 2)^{p}, R_{n+1}\right)}^{p} \leqslant \\
\leqslant\left\|\left(\bar{\theta}-k_{n}\right)_{-} \zeta_{n}\right\|_{p, Q\left((R / 2)^{p}, R_{n}\right)}^{p} \leqslant C\left\|\left(\bar{\theta}-k_{n}\right)_{-} \zeta_{n}\right\|_{V^{p}\left(Q\left((R / 2)^{p}, R_{n}\right)\right)}^{p} A_{n}^{p /(N+p)} \leqslant \\
\leqslant C \frac{2^{p n}}{(R / 2)^{p}}\left(\frac{\omega}{2^{s_{1}}}\right)^{p} A_{n}^{1+(p /(N+p))} .
\end{gathered}
$$

Next, define the numbers

$$
X_{n}=\frac{A_{n}}{\left|Q\left((R / 2)^{p}, R_{n}\right)\right|},
$$


divide the inequality by $\left|Q\left((R / 2)^{p}, R_{n+1}\right)\right|$ and obtain the recursive relation

$$
X_{n+1} \leqslant C 4^{p n} X_{n}^{1+(p /(N+p))} \text {. }
$$

Using again the lemma on the fast geometric convergence of sequences (see Lemma 4.1 on Chapter I of [6]) we conclude that if

$$
X_{0} \leqslant C^{-(N+p) / p} 4^{-(N+p)^{2} / p} \equiv v_{1} \in(0,1)
$$

then

$$
X_{n} \rightarrow \mathbf{0} .
$$

Apply Lemma 2 with this $v_{1}$ and conclude that there exists $s_{1}$, depending only on the data, and $\omega$ such that

$$
\left|\left\{x \in K_{R / 4}: \theta(x, t)<\mu_{-}+\frac{\omega}{2^{s_{1}}}\right\}\right| \leqslant v_{1}\left|K_{R / 4}\right|, \quad \forall t \in(-\hat{t}, 0),
$$

and obtain (34) as follows

$$
\begin{aligned}
X_{0} & =\frac{\int_{-(R / 2)^{p}}^{0} \int_{K_{R / 4}} \chi_{\left\{\left(\bar{\theta}-\left(\mu_{-}+\omega / 2^{s^{1}}\right)\right)_{-}>0\right\}}}{\left|Q\left((R / 2)^{p}, R / 4\right)\right|} \\
& =\frac{(R / 2)^{p}}{\hat{t}} \frac{\int_{-\hat{t}}^{0} \int_{K_{R / 4}} \chi_{\left\{\left(\theta-\left(\mu_{-}+\omega / 2^{s^{1}}\right)\right)_{-}>0\right\}}}{\left|Q\left((R / 2)^{p}, R / 4\right)\right|} \\
& \leqslant \frac{(R / 2)^{p}}{\hat{t}} \frac{\hat{t}\left|\left\{x \in K_{R / 4}: \theta(x, t)<\mu_{-}+\omega / 2^{s_{1}}\right\}\right|}{(R / 2)^{p}\left|K_{R / 4}\right|} \leqslant v_{1} .
\end{aligned}
$$

Now the conclusion easily follows from (35). In fact, observe that

$$
R_{n} \searrow \frac{R}{8} \quad \text { and } \quad k_{n} \searrow \mu_{-}+\frac{\omega}{2^{s_{1}+1}},
$$

and since (35) implies that $A_{n} \rightarrow 0$, we conclude that

$$
\begin{aligned}
\mid\left\{(x, \bar{t}) \in Q\left(\left(\frac{R}{2}\right)^{p}, \frac{R}{8}\right): \bar{\theta}(x, \bar{t})\right. & \left.\leqslant \mu_{-}+\frac{\omega}{2^{s_{1}+1}}\right\} \mid= \\
& =\left|\left\{(x, t) \in Q\left(\hat{t}, \frac{R}{8}\right): \theta(x, t) \leqslant \mu_{-}+\frac{\omega}{2^{s_{1}+1}}\right\}\right|=0
\end{aligned}
$$

and the proposition is proved. 
REMARK 4. - The dependence of $s_{1}$ on $\omega$ occurs only through $A$. Recalling the dependence of $A$ on $\omega$, that was already announced in (14), although it will only be determined in the next section, we find from (32) that it is sufficient to choose

$$
s_{1}>C 2^{C^{\prime} \omega^{-a}}>C_{1}+C_{2} 2^{C_{3}(p-2) \omega^{-\alpha}},
$$

that already takes into account the condition

$$
s_{1}>\log _{2} A+\frac{p}{p-2} \sim C \omega^{-\alpha}+\frac{p}{p-2} .
$$

CoRollary 1. - There exist constants $v_{0}, \sigma_{0} \in(0,1)$, depending on the data and $\omega$, such that if (17) holds then

$$
\underset{Q\left(d(R / 8)^{p}, R / 8\right)}{\operatorname{ess} O \leqslant \sigma_{0} \omega}
$$

Proof. - We can use Proposition 5 to obtain $s_{1} \in \mathbb{N}$ such that

$$
\underset{Q(i, R / 8)}{\operatorname{ess} \inf } \theta \geqslant \mu_{-}+\frac{\omega}{2^{s_{1}+1}}
$$

and from this we get

$$
\underset{Q(i, R / 8)}{\operatorname{ess}} \operatorname{osc} \theta=\underset{Q(i, R / 8)}{\operatorname{ess} \sup } \theta-\underset{Q(i, R / 8)}{\operatorname{ess} \inf } \theta \leqslant \mu_{+}-\mu_{-}-\frac{\omega}{2^{s_{1}+1}}=\left(1-\frac{1}{2^{s_{1}+1}}\right) \omega .
$$

Since $d(R / 8)^{p} \leqslant \hat{t}=-t^{*}+d(R / 2)^{p}, t^{*}<0$, we have

$$
Q\left(d\left(\frac{R}{8}\right)^{p}, \frac{R}{8}\right) \subset Q\left(\hat{t}, \frac{R}{8}\right)
$$

and the corollary follows with $\sigma_{0}=\left(1-1 /\left(2^{s_{1}+1}\right)\right)$.

\section{6. - Analysis of case (C2).}

If (C1) doesn't hold then (C2) is necessarily true. We will show that also in this case a conclusion similar to (37) can be taken. Recall that the constant $\nu_{0}$ has already been determined in the previous section and is given by (24).

Lemma 3. - Fix a cylinder $\left(0, t^{*}\right)+Q\left(d R^{p}, R\right) \subset Q\left(a_{0} R^{p}, R\right)$ for which (18) holds. There exists a time level

$$
t^{0} \in\left[t^{*}-d R^{p}, t^{*}-\frac{v_{0}}{2} d R^{p}\right]
$$


such that

$$
\left|\left\{x \in K_{R}: \theta\left(x, t^{0}\right)>\mu_{+}-\frac{\omega}{2}\right\}\right| \leqslant\left(\frac{1-v_{0}}{1-v_{0} / 2}\right)\left|K_{R}\right| .
$$

PRoof. - The proof can easily be obtained by contradiction (see Lemma 7.1. on Chapter III of [6]).

The next lemma asserts that the set where $\theta(x)$ is close to its supremum is small, not only at a specific time level, but for all time levels near the top of the cylinder $\left(0, t^{*}\right)+Q\left(d R^{p}, R\right)$.

Lemma 4. - There exists $1<s_{2} \in \mathbb{N}$, depending on the data and $\omega$, such that

$$
\left|\left\{x \in K_{R}: \theta(x, t)>\mu_{+}-\frac{\omega}{2^{s_{2}}}\right\}\right| \leqslant\left(1-\left(\frac{\nu_{0}}{2}\right)^{2}\right)\left|K_{R}\right|,
$$

for all $t \in\left[t^{*}-\left(\nu_{0} / 2\right) d R^{p}, t^{*}\right]$.

Proof. - The proof consists in using the logarithmic inequalities of Proposition 4, and since these are the same as in the nonsingular case, the proof is the same as in Lemma 7.2 on Chapter III of [6]. We just trace the dependence of $s_{2}$ on $\omega$. The number $s_{2}$ is chosen in [6], $s_{2}=n+1$ where $\mathrm{n}$ is chosen so large that

$$
\left(\frac{n}{n-1}\right)^{2} \leqslant\left(1-\frac{v_{0}}{2}\right)\left(1+v_{0}\right) \equiv \beta \quad \text { and } \quad n \geqslant C v_{0}^{-2(p+1)} \text {. }
$$

Assuming $v_{0} \leqslant 1 / 2$, which is always possible, we conclude that

$$
\frac{4}{v_{0}^{2}}+2 \geqslant \frac{4}{v_{0}\left(1-v_{0}\right)}+2=\frac{2 \beta}{\beta-1}>\frac{\beta+\sqrt{\beta}}{\beta-1},
$$

since $\beta>1$, so it is sufficient to choose

$$
n>\max \left\{\frac{4}{v_{0}^{2}}+2, C v_{0}^{-2(p+1)}\right\} .
$$

Recalling the dependence of $\nu_{0}$ on $\omega$, it is sufficient to choose

$$
s_{2}>\frac{C}{\omega^{2(p+1)(N+p) / p}}=C \omega^{-\alpha} .
$$

Now we want to show that the same type of conclusion holds in an upper portion of the full cylinder $Q\left(a_{0} R^{p}, R\right)$, say for all $t \in\left(-\left(a_{0} / 2\right) R^{p}, 0\right)$. We just have to use the fact that (18) holds for all cylinders of the type $\left(0, t^{*}\right)+Q\left(d R^{p}, R\right)$ so that the conclu- 
sion of the previous lemma holds true for all time levels

$$
t \geqslant-\left(a_{0}-d\right) R^{p}-\frac{\nu_{0}}{2} d R^{p} .
$$

So if we choose

$$
\left(\frac{A}{2}\right)^{p-2} \geqslant 2
$$

we get

$$
\frac{a_{0}}{d} \geqslant 2-\nu_{0} \Leftrightarrow-\left(a_{0}-d\right) R^{p}-\frac{\nu_{0}}{2} d R^{p} \leqslant-\frac{a_{0}}{2} R^{p}
$$

and obtain

CoRollary 2. - For all $t \in\left(-\left(a_{0} / 2\right) R^{p}, 0\right)$,

$$
\left|\left\{x \in K_{R}: \theta(x, t)>\mu_{+}-\frac{\omega}{2^{s_{2}}}\right\}\right| \leqslant\left(1-\left(\frac{\nu_{0}}{2}\right)^{2}\right)\left|K_{R}\right| .
$$

This information can be used to prove the main result of this section.

Proposition 6. - There exists a constant $1<s_{3} \in \mathbb{N}$, depending on the data and $\omega$, such that

$$
\theta(x, t) \leqslant \mu_{+}-\frac{\omega}{2^{8_{3}+1}} \quad \text { a.e. }(x, t) \in Q\left(\frac{a_{0}}{2}\left(\frac{R}{2}\right)^{p}, \frac{R}{2}\right)
$$

Proof. - Again the proof is the same as in Lemma 9.1 of Chapter III of [6]. We trace here the dependence of $s_{3}$ on $\omega$. In [6], $s_{3}$ is determined using an auxiliary lemma (Lemma 8.1 on Chapter III) that says that given any $v_{3} \in(0,1)$, there exists $s_{3}>s_{2}$ such that

$$
\left|\left\{(x, t) \in Q\left(\frac{a_{0}}{2} R^{p}, R\right): \theta(x, t)>\mu_{+}-\frac{\omega}{2^{s_{3}}}\right\}\right| \leqslant v_{3}\left|Q\left(\frac{a_{0}}{2} R^{p}, R\right)\right| .
$$

The $v_{3}$ used to determine $s_{3}$ depends only on the data and the choice of $s_{3}$ is made so that

$$
\frac{C}{v_{0}^{2}\left(s_{3}-s_{2}\right)^{(p-1) / p}} \leqslant v_{3}
$$


that is

$$
s_{3}>s_{2}+\frac{C}{v_{0}^{2 p /(p-1)}} \sim \frac{C_{1}}{\omega^{2(p+1)(N+p) / p}}+\frac{C_{2}}{\omega^{2(N+p) /(p-1)}} .
$$

It is clearly enough to choose

$$
s_{3}>\frac{C}{\omega^{2(p+1)(N+p) / p}}=C \omega^{-\alpha} .
$$

It is still here that $A$ is chosen equal to

$$
A=2^{s_{3}},
$$

( $s_{3}$ so large that (38) holds) which also determines the length of the cylinder $Q\left(a_{0} R^{p}, R\right)$. Observe that in this way we determine a level $\mu_{+}-\omega / 2^{s_{3}+1}$ and a cylinder (fixing $A$ and consequently $a_{0}$ ) so that (39) holds in that particular cylinder.

CoROLLARY 3. - There exist constants $v_{0}, \sigma_{1} \in(0,1)$, depending on the data and $\omega$, such that if (18) holds then

$$
\underset{Q\left(a_{0} / 2(R / 2)^{p}, R / 2\right)}{\operatorname{ess}} \theta \leqslant \sigma_{1} \omega
$$

Proof. - It is similar to the proof of Corollary 1. We find $\sigma_{1}=\left(1-1 / 2^{s_{3}+1}\right)$.

\section{7. - Proof of the main results.}

In this section we prove our main results using the the information obtained previously. An immediate consequence of Corollaries 1 and 3 is

LEMma 5. - There exists a constant $\sigma=\sigma(\omega) \in(0,1)$, that depends only on the data and $\omega$, such that

$$
\underset{Q\left(d(R / 8)^{p}, R / 8\right)}{\operatorname{ess} \operatorname{osc}} \theta \leqslant \sigma(\omega) \omega
$$

Proof. - Since one of (17) or (18) has to be true, the conclusion of at least one of Corollaries 1 or 3 holds. Choosing

$$
\sigma=\max \left\{\sigma_{0}, \sigma_{1}\right\},
$$

and observing that, due to (38),

$$
d\left(\frac{R}{8}\right)^{p} \leqslant \frac{a_{0}}{2}\left(\frac{R}{2}\right)^{p},
$$

we obtain the conclusion. 

Set

We are now going to define recursively two sequences of positive real numbers.

$$
\omega_{1}=\sigma(\omega) \omega \quad \text { and } \quad R_{1}=\frac{R}{\kappa\left(\omega_{1}\right)}
$$

with

$$
\kappa\left(\omega_{1}\right)=8\left(\frac{1}{\sigma(\omega)}\right)^{(p-2) / p}\left(\frac{A\left(\omega_{1}\right)}{2}\right)^{p-2 / p}>8,
$$

$A\left(\omega_{1}\right)$ to be fixed depending on $\omega_{1}$. Then defining

$$
Q_{1}=Q\left(a_{1} R_{1}^{p}, R_{1}\right) \quad \text { with } \quad a_{1}=\left(\frac{\omega_{1}}{A\left(\omega_{1}\right)}\right)^{2-p},
$$

and since

$$
\begin{aligned}
a_{1} R_{1}^{p} & =\left(\frac{\omega_{1}}{A\left(\omega_{1}\right)}\right)^{2-p}\left(\frac{1}{\sigma(\omega)}\right)^{2-p}\left(\frac{A\left(\omega_{1}\right)}{2}\right)^{2-p}\left(\frac{R}{8}\right)^{p} \\
& =\left(\frac{\omega_{1}}{A\left(\omega_{1}\right)}\right)^{2-p}\left(\frac{\omega}{\omega_{1}}\right)^{2-p}\left(\frac{A\left(\omega_{1}\right)}{2}\right)^{2-p}\left(\frac{R}{8}\right)^{p} \\
& =\left(\frac{\omega}{2}\right)^{2-p}\left(\frac{R}{8}\right)^{p} \\
& =d\left(\frac{R}{8}\right)^{p},
\end{aligned}
$$

we have

$$
Q_{1} \subset Q\left(a_{0} R^{p}, R\right) \quad \text { and } \quad \underset{Q_{1}}{\operatorname{ess}} \operatorname{osc} \theta \leqslant \omega_{1} .
$$

We are in the setting (16) and this means that the whole process can now be repeated starting from $Q_{1}$. So we define recursively the following sequences of positive real numbers, for $n=0,1, \ldots$,

$$
\left\{\begin{array} { l } 
{ R _ { 0 } = R } \\
{ R _ { n + 1 } = \frac { R _ { n } } { \kappa ( \omega _ { n + 1 } ) } }
\end{array} \text { and } \left\{\begin{array}{l}
\omega_{0}=\omega \\
\omega_{n+1}=\sigma\left(\omega_{n}\right) \omega_{n}
\end{array}\right.\right.
$$


and consider the family of cylinders

$$
Q_{n}=Q\left(a_{n} R_{n}^{p}, R_{n}\right), \quad \text { with } \quad a_{n}=\left(\frac{\omega_{n}}{A\left(\omega_{n}\right)}\right)^{2-p}
$$

THEOREM 2. - The sequences $\left(\omega_{n}\right)_{n}$ and $\left(R_{n}\right)_{n}$ are decreasing sequences that converge to zero. Moreover, for all $n=0,1,2, \ldots$,

$$
Q_{n+1} \subset Q_{n} \quad \text { and } \quad \underset{Q_{n}}{\operatorname{ess}} \operatorname{osc} \theta \leqslant \omega_{n} .
$$

Proof. - The sequences are obviously decreasing and bounded below by zero, so to show that they converge to zero we just need to show that they cannot converge to a positive number. As far as $\left(R_{n}\right)_{n}$ is concerned this conclusion follows immediately from

$$
\frac{R_{n+1}}{R_{n}}=\frac{1}{\kappa\left(\omega_{n+1}\right)}<\frac{1}{8} .
$$

With $\left(\omega_{n}\right)_{n}$ the situation is more delicate since

$$
\frac{\omega_{n+1}}{\omega_{n}}=\sigma\left(\omega_{n}\right)>1
$$

So we suppose that $\omega_{n} \searrow \alpha>0$ and observe that, in that case,

$$
\sigma\left(\omega_{n}\right) \nearrow \sigma(\alpha)<1
$$

recalling that

$$
\sigma\left(\omega_{n}\right)=1-\frac{1}{2^{s+1}}, \quad s=s\left(\omega_{n}\right)=\max \left\{s_{1}\left(\omega_{n}\right), s_{3}\left(\omega_{n}\right)\right\}
$$

and the dependence of $s_{1}$ and $s_{3}$ on $\omega_{n}$, given respectively by (36) and (40). Consequently, $\omega_{n+1}=\sigma\left(\omega_{n}\right) \omega_{n} \leqslant \sigma(\alpha) \omega_{n}$ and

$$
\omega_{n} \leqslant[\sigma(\alpha)]^{n} \omega, \quad n=0,1, \ldots,
$$

which implies that $\omega_{n} \rightarrow 0$, a contradiction.

Relations (41) follow at once from the recursive process used to define the sequences.

Theorem 2 was obtained assuming (15). If this doesn't hold, we have, recalling the dependence of $A$ on $\omega$ given by (14),

$$
\left(\frac{\omega}{A}\right)^{p-2} \leqslant R \Rightarrow \frac{1}{2^{C \omega^{-\alpha}}} \leqslant \frac{\omega}{2^{C \omega-\alpha}} \leqslant R^{1 /(p-2)},
$$

since $\omega<1$. Now, taking logarithms and assuming without loss of generality that $R<$ 
$<1$, we obtain

$$
C^{\prime} \omega^{-\alpha} \geqslant-\log _{2} R^{1 /(p-2)} \Rightarrow \omega \leqslant\left\{\frac{C^{\prime}}{-\log _{2} R^{1 /(p-2)}}\right\}^{1 / \alpha} \rightarrow 0, \quad R \rightarrow 0,
$$

and there is nothing to prove.

Proof of Theorem 1. - The conclusion of Theorem 2 implies that

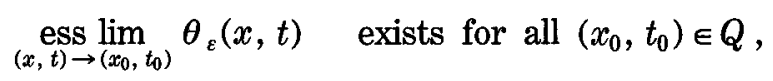

so we can choose a continuous representative for each $\theta_{\varepsilon}$ out of its equivalence class. It also defines implicitly an interior modulus of continuity, i.e., for each $K \subset Q$, a continuous and nondecreasing function $f_{K}: \mathbb{R}^{+} \rightarrow \mathbb{R}^{+}$, that depends only on the data and $K$, such that

$$
\left|\theta_{\varepsilon}(x, t)-\theta_{\varepsilon}\left(x^{\prime}, t^{\prime}\right)\right| \leqslant f_{K}\left(\left|x-x^{\prime}\right|+\left|t-t^{\prime}\right|^{1 / p}\right) .
$$

Since this modulus of continuity is independent of $\varepsilon$ (cf. Remark 3), the conclusion of the theorem follows.

REMARK 5. - The results can be extended up to the parabolic boundary of $Q$. In fact, we can obtain a continuous solution at $t=0$ and at the boundary $\Sigma$, in the case of Neumann or homogeneous Dirichlet data. The proof consists in adapting the energy and logarithmic estimates and the proof of the interior continuity as in [3] and [6]. The case of nonhomogeneous Dirichlet data presented unexpected difficulties in the case $p=2$, dealt with at [5], and is left open in the case $p>2$.

REMARK 6. - Also the principal part singular case $1<p<2$ is left open. Apart from the extra difficulties that may arise from the more elaborate techniques that have to be applied, there is also a problem concerning the regularization that is used in [11] to obtain the solution for $1<p<2$. It consists in adding a perturbation of the Laplacian to the equation, i.e., an extra term of the form

$$
\varepsilon \int_{Q} \nabla \theta_{\varepsilon} \cdot \nabla \xi
$$

to (4) and this is clearly a bad term as far as the derivation of independent of $\varepsilon$ energy and logarithmic estimates is concerned. So we should first try to obtain a solution using a different, and more adequate for this purpose, type of regularization. We expect to overcome these difficulties in the future.

Acknowledgments. This work was concluded during a visit to the University of Rome II "Tor Vergata». I would like to thank Prof. Emmanuele DiBenedetto for the hospitality and the fruitful suggestions and remarks concerning the article. 
This work is part of my Ph.D. thesis elaborated at C.M.A.F. / University of Lisbon. I would like to thank my advisor Prof. José Francisco Rodrigues for all of his help and encouragement. I also acknowledge the excellent research atmosphere at C.M.A.F.

\section{REFERENCES}

[1] L. A. CAFFarelli - L.C. Evans, Continuity of the temperature in the two phase Stefan problem, Arch. Ration. Mech. Anal., 81 (1983), pp. 199-220.

[2] E. DeGioRgI, Sulla differenziabilitá e l'analiticitá delle estremali degli integrali multipli regolari, Mem. Accad. Sci. Torino, Cl. Sci. Fis. Mat. Nat., Ser. 3, 3 (1957), pp. 25-43.

[3] E. DiBenedetTo, Continuity of weak solutions to certain singular parabolic equations, Ann. Mat. Pura Appl. (IV), 130 (1982), pp. 131-176.

[4] E. DiBenEDETTo, Continuity of weak solutions to a general porous medium equation, Indiana Univ. Math. J., 32, 1 (1983), pp. 83-118.

[5] E. DiBenEDETTO, A boundary modulus of continuity for a class of singular parabolic equations, J. Differ. Equations, 63 (1986), pp. 418-447.

[6] E. DiBenedetto, Degenerate Parabolic Equations, Springer-Verlag, New York, 1993.

[7] S. Kamenomostkaya, On the Stefan problem (in Russian), Naučnye Dokl. Vysšei Školy, 1 (1958), pp. 60-62; Mat. Sb., 53 (95) (1961), pp. 489-514.

[8] O. LADYZENSKAJA - V. SOLONNIKOV - N. URAL'CEVA, Linear and Quasi-linear Equations of Parabolic Type, A.M.S. Transl. Monog. 23, Providence, R.I., 1968.

[9] J. Moser, $A$ new proof of DeGiorgi's theorem concerning the regularity problem for elliptic differential equations, Comm. Pure Appl. Math., 13 (1960), pp. 457-468.

[10] J. F. RodRIGUES, Variational methods in the Stefan problem, in: Phase Transitions and Hysteresis (A. Visintin, Ed.), pp. 147-212, Lect. Notes Math. 1584, Springer-Verlag, Berlin, 1994.

[11] J. M. URBANo, A free boundary problem with convection for the p-Laplacian, Rend. Mat. Appl. (VII), 17 (1997), pp. 1-19. 